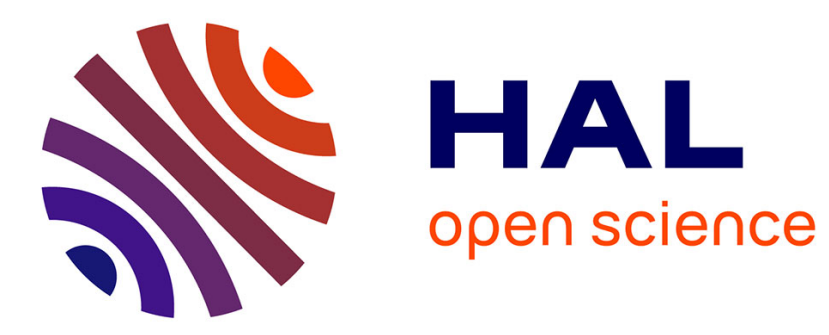

\title{
La recomposition des solidarités entre agriculteurs aux Pays-Bas : écologisation des pratiques ou transformations managériales?
}

François-Joseph Daniel

\section{- To cite this version:}

François-Joseph Daniel. La recomposition des solidarités entre agriculteurs aux Pays-Bas: écologisation des pratiques ou transformations managériales?. Revue d'Etudes en Agriculture et Environnement - Review of agricultural and environmental studies, 2012, 93 (1), pp.31-47. 10.4074/S1966960712001026 . hal-00939065

\section{HAL Id: hal-00939065 https://hal.science/hal-00939065}

Submitted on 30 Jan 2014

HAL is a multi-disciplinary open access archive for the deposit and dissemination of scientific research documents, whether they are published or not. The documents may come from teaching and research institutions in France or abroad, or from public or private research centers.
L'archive ouverte pluridisciplinaire HAL, est destinée au dépôt et à la diffusion de documents scientifiques de niveau recherche, publiés ou non, émanant des établissements d'enseignement et de recherche français ou étrangers, des laboratoires publics ou privés. 


\title{
La recomposition des solidarités entre agriculteurs aux Pays-Bas : écologisation des pratiques ou transformations managériales?
}

\author{
François-Joseph DANIEL \\ Ecole nationale du génie de l'eau et de l'environnement de Strasbourg (ENGEES), UMR GESTE, \\ 1 quai Koch, BP 61039, 67070 Strasbourg cedex, France \\ e-mail : daniel.francois-joseph@engees.unistra.fr
}

Résumé - Face à la montée en puissance des réglementations environnementales, les formes de solidarité entre les agriculteurs professionnels font l'objet, depuis une vingtaine d'années, d'importantes recompositions. Aux Pays-Bas, ces recompositions ont donné lieu à l'émergence de nouvelles organisations professionnelles locales - les « coopératives environnementales ». Ces transformations s'expliquent tant par des dynamiques d'écologisation des pratiques, qui poussent les organisations agricoles à revoir leur projet fondateur, que par les réformes managériales de l'Etat, qui favorisent la construction de nouvelles logiques associatives entre professionnels. Ces formes de solidarités, induites par les politiques interventionnistes de gestion de la nature, marquent un tournant historique dans la gouvernance du secteur. Elles témoignent d'un recul de l'autonomie professionnelle et de l'instauration d'un «professionnalisme de l'extérieur » au sein duquel les professionnels ont moins de prises sur les conditions d'exercice de leur métier.

Mots-clés : écologisation, coopérative environnementale, agriculture, solidarité, groupe professionnel, autonomie professionnelle, Pays-Bas, nouvelle gestion publique

\section{Restructuring farmers' solidarities in The Netherlands: Ecologising of practices or public management reforms? \\ Summary - In light of the powerful increase in environmental regulations in the past 20 years, the solidarities among professional farmers are undergoing important reforms. In the Netherlands, these restructurings bave given rise to new locally-based organizations - the "environmental cooperatives". These transformations can be explained both by the dynamic of ecologising the practices that encourage agricultural organizations to review their initial projects, and by the State's public management reforms that stimulate the construction of new underlying principles among professional associations. These new forms of associative unity, induced by the nature protection policies, mark a bistorical shift in the management of the agricultural sector. They attest to a certain decrease in professional autonomy, and to the institution of a professionnalism constructed "from above" at the heart of which the farmers have less control over their working conditions.}

Keywords: ecologising, environmental cooperative, agriculture, solidarity, professional group, professional autonomy, the Netherlands, New Public Management

Classification JEL : Q56, Y80

\section{Remerciements}

Je tiens à remercier les deux relecteurs anonymes pour leurs commentaires constructifs qui ont permis d'améliorer ce texte. 


\section{Introduction}

Aux Pays-Bas, comme dans bien d'autres pays européens, le groupe professionnel agricole connaît, depuis plus d'une vingtaine d'années, de fortes tensions liées à la remise en cause du modèle productiviste qui avait fait le succès des années de modernisation (Van der Ploeg, 2003). Cette remise en question a profondément transformé le projet des organisations professionnelles qui ont, au cours des deux dernières décennies, réajusté leur discours et restructuré en profondeur leurs projets fondateurs. Si ce réajustement a été analysé dans une large mesure du point de vue de "l'écologisation» des pratiques (Kalaora, 2001 ; Deverre et de Sainte Marie, 2008), la question concomitante, et tout aussi significative, des évolutions du mode de gouvernance du secteur et de ses implications sur le groupe professionnel mérite d'être posée. Certes, le contenu du «mandat» s'est transformé - d'une agriculture productiviste à un modèle professionnel intégrant dans ses modes de production les dimensions environnementales -, mais le mode de régulation de la profession a été lui aussi l'objet de profondes transformations. Alors que la modernisation agricole s'est historiquement structurée autour d'une collaboration très forte entre agriculteurs et pouvoirs publics dans la plupart des pays d'Europe occidentale (Hervieu et Lagrave, 1992), les enjeux de développement durable marquent une épreuve dans la nature du «contrat implicite» qui lie l'Etat et la profession. Cette mise à l'épreuve est assurément liée à la relative perte de légitimité des professionnels agricoles face à leur incapacité à gérer les ressources environnementales, mais pas uniquement. Les évolutions «managérialistes » des modes de gouvernance du secteur agricole ont en effet contribué à transformer profondément la structure des organisations professionnelles. Comme le soulignent Boussard et al. (2010), ces évolutions qui touchent un certain nombre de professions dites réglementées ont toutes en commun la révision de leur mandat et de l'autonomie leur étant attribuée pour l'accomplir. Les réformes managériales des administrations publiques placent en effet les professions en situation de rendre des comptes sur l'efficacité de leur travail. Elles ont mis à l'ordre du jour des principes d'obligation de résultat, d'évaluation et de contrôle dans la gestion des affaires publiques (Pollitt et Bouckaert, 2000; De Visscher et Varone, 2004 ; Bezes, 2008). En organisant la gouvernance extra-professionnelle des pratiques, ces logiques gestionnaires ont réduit la capacité des groupes professionnels à dire ce qu'est une bonne pratique - d'où l'assimilation de ces logiques par certains chercheurs à un processus de «déprofessionnalisation» (Demailly et de la Broise, 2009). Ce recul de «l'autonomie professionnelle (Boussard et al., 2010) n'est pas sans impacter des organisations professionnelles elles-mêmes, leur projet fondateur et la nature des solidarités qui structurent la cohésion de ces groupes. Aux Pays-Bas, face à la montée des réglementations environnementales et aux transformations managériales de l'Etat, le groupe professionnel agricole a non seulement vu son autonomie de régulation se restreindre, mais il a aussi fait l'objet d'une profonde restructuration. Le réajustement $\mathrm{du}$ projet des organisations professionnelles agricoles à l'égard des considérations d'ordre écologique et environnemental a été associé à une recomposition importante du paysage organisationnel: l'apparition d'une nouvelle génération d'organisations agricoles appelées « coopératives environnementales » en est le signe le plus frappant. L'ambition de cet article est de mettre en lumière ces recompositions en interrogeant 
les formes de solidarités à l'œuvre entre professionnels à partir de l'examen des projets de ces coopératives et de leur l'évolution dans le temps. L'objectif n'est pas de retracer l'histoire exhaustive de ces organisations, ni même d'exposer dans le détail leur contexte d'émergence et les stratégies de légitimation qu'elles ont mises en place (ce qui a été fait par ailleurs, of. Van der Ploeg et Renting, 2001; Daniel, 2011a et 2011b), mais bien d'exposer la complexité du projet coopératif et l'impact des transformations managériales sur l'évolution de ce projet. Nous reviendrons, d'abord, sur la notion de solidarité qui nous intéresse ici et, plus particulièrement, sur la façon dont elle s'est historiquement incarnée au sein du groupe professionnel agricole. Puis nous présenterons brièvement le phénomène des coopératives environnementales, les enjeux de leur émergence et les interprétations qui ont été faites de ce phénomène. Enfin, nous exposerons les différentes logiques à travers lesquelles les projets associatifs se sont incarnés.

\section{Les évolutions de la solidarité entre agriculteurs professionnels}

\subsection{Les liens de solidarités au sein du groupe professionnel agricole}

La notion de solidarité est entendue ici dans son acception durkheimienne (Durkheim, 2007 [1893]). Elle correspond à l'ensemble des liens de réciprocité qui, dans une organisation, marquent la stabilité des liens entre des individus et permettent à un projet collectif de s'inscrire dans la durée. La solidarité ne correspond pas seulement aux motifs invoqués par les individus dans la construction d'un collectif. Elle renvoie aussi aux actions concrètes qui matérialisent et incarnent l'existence de ces liens, en somme, ce qui est constructeur de liens entre les individus qui s'engagent dans l'action. La définition de la solidarité que nous mobilisons pour décrire les liens de réciprocités entre agriculteurs est donc beaucoup plus large que celle habituellement utilisée dans le cadre de l'économie sociale et solidaire, dans la mesure où elle ne renvoie pas uniquement à un secteur de l'économie. Comme le souligne Zoll (1998) en reprenant la distinction avancée par Durkheim entre solidarité organique et mécanique, la structuration des groupes professionnels s'organise plus autour de solidarités fondées sur la ressemblance, l'égalité et l'appartenance à une même communauté (solidarités mécaniques), qu'autour de solidarités fondées sur la différence et le dépassement des frontières de la communauté (solidarités organiques). Les formes de solidarités entre membres du groupe professionnel reposent en effet davantage sur des enjeux de cohésion interne que sur des enjeux de mise en relation des groupes sociaux confrontés à des problématiques sociétales transversales.

En agriculture, aux Pays-Bas comme en France, ces liens de réciprocité semblent s'être historiquement structurés autour de différents projets professionnels. Le premier de ces projets a été orienté vers la gestion collective du risque de marché avec l'essor au $\mathrm{XX}^{\mathrm{e}}$ siècle du mouvement coopératif et mutualiste. Mutualiser les approvisionnements, assurer les risques de mauvaises récoltes, coordonner la vente et la transformation des produits agricoles, etc. ont été autant de mesures mises en place au sein d'organisations professionnelles diverses pour assurer la maîtrise collective des marchés agricoles (Devienne, 1989). Les agriculteurs ont bien sûr également mis sur pied des 
organisations de représentation syndicale et politique du groupe professionnel. Ce projet multipolaire s'est structuré autour de la défense non seulement des intérêts agricoles, mais aussi d'une cause ou d'une vision du métier - celle du statut d'entrepreneur a très fortement servi de mythe mobilisateur pendant la période de modernisation de l'agriculture (Van der Ploeg, 2003). Les agriculteurs ont également contribué à l'effort de diffusion du projet technique par le biais de puissantes organisations professionnelles qui, en collaboration avec l'Etat, ont permis de propager les innovations nécessaires à la modernisation des structures d'exploitation (Labarthe, 2006). Enfin, les organisations agricoles ont fortement renforcé leur projet professionnel à travers les mandats donnés par les pouvoirs publics dans la gestion administrative de certaines missions (gestion du foncier, de la modernisation des structures, des dossiers d'installation, etc.) (Devienne, 1989). Les organisations professionnelles, de par leur rôle dans la modernisation de l'agriculture et leur reconnaissance par les pouvoirs publics, ont fortement structuré le groupe professionnel et cristallisé les liens de solidarité entre les agriculteurs.

\subsection{La solidarité entre professionnels agricoles à l'épreuve de l'environnement}

A partir des années 1980, la crise du modèle productiviste a fortement mis à l'épreuve le groupe professionnel néerlandais, son projet collectif et le rapport qu'il entend entretenir avec les autres groupes de la société. C'est dans un contexte d'instabilité marqué par la libéralisation de la PAC et la montée en puissance des réglementations environnementales que le groupe professionnel agricole a été amené à se transformer profondément à partir des années 1990. A l'instar de nombreux groupes professionnels en situation de fortes tensions institutionnelles (Demazière et Gadéa, 2009), certains agriculteurs, parmi les plus favorables à une appropriation offensive du discours environnementaliste, ont réajusté leur projet coopératif en créant de nouvelles organisations agricoles locales, appelées « coopératives environnementales » ${ }^{1}$.

Les économistes néo-institutionnels ont été parmi les premiers à donner une interprétation des logiques d'association existantes entre ces agriculteurs. Selon eux, les coopératives environnementales sont des organisations dont la vocation est de pallier les défaillances du marché en matière de gestion durable des biens publics environnementaux (Hagedorn et al., 2002; Polman et Slangen, 2002; Slangen et Polman, 2002). Ces organisations permettraient non seulement de rendre optimale l'organisation de la gestion de la nature en se présentant comme une alternative aux mécanismes marchands, mais aussi de maximiser, par un engagement volontaire, les intérêts individuels des agriculteurs y participant. Non seulement cette interprétation reprend le mythe mobilisateur du projet agricole, mais elle semble également introduire un présupposé optimaliste dans la mesure où les coopératives environnementales sont vues comme la réponse institutionnelle la plus efficace aux problèmes d'environnement. L'engagement dans la coopération est ainsi présenté

\footnotetext{
${ }^{1}$ Le terme de coopérative (cooperatie en néerlandais) est utilisé dans une acception plus large que celle communément admise dans le cadre de l'économie sociale et solidaire. Il correspond à une forme d'«agir ensemble». Certaines de ces organisations se font d'ailleurs également appeler «associations » (vereniging).
} 
comme un choix rationnel destiné à maximiser la gestion des biens environnementaux. De plus, la forme organisationnelle prise par ce mouvement agricole semble naturalisée à un niveau local comme si la nature territorialisée de ces organisations constituait une donnée inflexible. Peut-on toutefois considérer que ces recompositions organisationnelles ne peuvent se matérialiser que dans un tel cadre ou bien celui-ci est-il lui-même le résultat d'un construit social situé dans le temps et dans l'espace?

Ces interprétations nous interrogent sur la réalité des liens de solidarité qui associent les agriculteurs professionnels les uns aux autres. Pour mieux comprendre la nature de ces liens et la façon dont ils ont émergé, il nous a semblé nécessaire d'adopter une perspective analytique nous amenant à considérer ces organisations comme le résultat d'une construction sociale et non d'une forme organisationnelle allant de soi, d'un ordre naturel nécessaire. Des travaux précédents ont montré que les agriculteurs engagés dans ces organisations se sont positionnés dans des logiques de résistance face à l'essor des réglementations environnementales et aux épisodes de libéralisation de la $\mathrm{PAC}^{2}$. Leurs démarches de contre-mobilisation se sont structurées autour d'une stratégie d'ouverture du «territoire professionnel » à des acteurs non agricoles (Daniel, $2011 \mathrm{~b}$ ), et d'un discours d'auto-gouvernance locale en partie co-généré par des chercheurs en sociologie rurale qui ont, dans le cadre d'un processus performatif de recherche action, contribué à mettre en cohérence ce réajustement discursif (Daniel, 2011a). Ces recherches ont jusqu'à présent peu mis en évidence les processus par lesquels ces organisations se sont généralisées sur l'ensemble du territoire.

L'exploitation d'une enquête réalisée entre 2004 et 2006 nous a permis d'approfondir la logique de diffusion de cette innovation organisationnelle. Cette enquête comprend un examen de la littérature existante sur les coopératives environnementales ainsi qu'une quarantaine d'entretiens retranscrits et analysés auprès d'agriculteurs membres de coopératives, de responsables et d'employés des fédérations régionales et nationales de coopératives, de personnes plus ou moins impliquées dans le mouvement (personnalités politiques locales, représentants d'organisations environnementales ou de protection de l'environnement, employé municipal, etc.), et de responsables de services administratifs provinciaux et nationaux en charge des questions agroenvironnementales. Nous avons veillé à couvrir la diversité des coopératives en prenant en compte l'âge de ces organisations, leur situation géographique et leurs caractéristiques agronomiques (types de production, sols). Ce travail nous a permis de mettre en lumière les formes de solidarités qui y ont été

\footnotetext{
${ }^{2}$ Les premières coopératives environnementales proviennent de groupes locaux d'agriculteurs mobilisés contre l'application d'une succession de lois environnementales, européennes et nationales, qui se sont multipliées à la fin des années 1980. Ces agriculteurs se sont opposés, en fonction des enjeux auxquels ils ont été confrontés sur leur territoire, aux lois environnementales encadrant les effluents d'élevage dans les zones de forte production animale, à la forte pression exercée par l'urbanisation ou encore aux projets gouvernementaux de reconquête des espaces de « nature » sur les espaces agricoles (De Bruin et Van Der Ploeg, 1991; Van der Ploeg et Renting, 2001 ; Luttik et Van der Ploeg, 2004 ; Boonstra, 2006, p. 59; Padt, 2007, p. 138). Dans chacun des cas, ces initiatives ont pris forme en réponse à la montée en puissance d'un cadre réglementaire jugé contraignant par les agriculteurs qui y voyaient une limite à leurs capacités d'expansion économique dans un contexte de libéralisation des politiques agricoles (Daniel, 2011b).
} 
construites, les raisons pour lesquelles celles-ci se sont incarnées dans des formes d'organisations agricoles localisées, les effets de ces projets associatifs sur le groupe professionnel et les difficultés rencontrées par les instigateurs de ces initiatives.

Tableau 1. Liste des coopératives analysées

\begin{tabular}{|c|c|c|c|c|c|}
\hline $\begin{array}{l}\text { Nom de la coopérative } \\
\text { environnementale }\end{array}$ & $\begin{array}{l}\text { Production } \\
\text { dominante }\end{array}$ & $\begin{array}{l}\text { Estimation } \\
\text { du nombre } \\
\text { d'adhérents* }\end{array}$ & $\begin{array}{c}\text { Situation } \\
\text { géographique } \\
\text { (province) }\end{array}$ & $\begin{array}{c}\text { Type } \\
\text { de sol** }\end{array}$ & $\begin{array}{l}\text { Année } \\
\text { de } \\
\text { création }\end{array}$ \\
\hline $\begin{array}{l}\text { Vereniging Eastermar's } \\
\text { Lansdouwe (VEL) }\end{array}$ & $\begin{array}{l}\text { Elevages } \\
\text { laitiers }\end{array}$ & Plus de 100 & Friesland & Sablonneux & 1992 \\
\hline Achtkarspelen (VANLA) & $\begin{array}{l}\text { Elevages } \\
\text { laitiers }\end{array}$ & Plus de 100 & Friesland & Sablonneux & 1992 \\
\hline $\begin{array}{l}\text { Milieucoöperatie PION- } \\
\text { De Peel }\end{array}$ & $\begin{array}{l}\text { Production } \\
\text { porcine }\end{array}$ & Plus de 100 & Noord-Brabant & Sablonneux & 1996 \\
\hline Ecolana & $\begin{array}{l}\text { Céréaliculture/ } \\
\text { production } \\
\text { laitière }\end{array}$ & Moins de 10 & Friesland & Argileux & 2001 \\
\hline $\begin{array}{l}\text { Coöperatie } \\
\text { Rivierduingebied }\end{array}$ & $\begin{array}{l}\text { Elevages } \\
\text { laitiers }\end{array}$ & Entre 10 et 100 & Flevoland & Sablonneux & 2000 \\
\hline De Kop van de NOP & Céréaliculture & Moins de 10 & Flevoland & Argileux & 2001 \\
\hline Zwartemeerdijk & Céréaliculture & Moins de 10 & Flevoland & Argileux & 2002 \\
\hline Waterland & $\begin{array}{l}\text { Elevages } \\
\text { laitiers }\end{array}$ & Plus de 100 & Noord Holland & $\begin{array}{l}\text { Argileux - } \\
\text { Tourbeux }\end{array}$ & 1996 \\
\hline Den Hâneker & $\begin{array}{l}\text { Elevages } \\
\text { laitiers }\end{array}$ & Plus de 100 & Zuid Holland & Argileux & 1994 \\
\hline Koningsdiep & $\begin{array}{l}\text { Elevages } \\
\text { laitiers }\end{array}$ & Entre 10 et 100 & Friesland & Tourbeux & 1996 \\
\hline
\end{tabular}

Notes : * Ces estimations s'appuient sur l'enquête réalisée en 2004 par Oerlemans et al. (2004).

** Aux Pays-Bas, les sols argileux témoignent en général d'une activité agricole présente sur les polders, c'est-à-dire des terres agricoles très productives d'un point de vue agronomique ayant été gagnées sur la mer ou situées en proximité des cours d'eau.

Trois logiques associatives ont été identifiées: des logiques de représentation, d'innovation sociotechnique et de gestion. Ces trois logiques associatives (qui ne sont pas nécessairement et systématiquement présentes dans chacune des organisations) s'articulent peu ou prou les unes avec les autres au sein des projets. Leur apparition dans le temps marque une évolution significative du projet coopératif agricole néerlandais.

\section{Les transformations de la représentation des agriculteurs ou " comment mieux défendre les intérêts agricoles?"}

Le projet coopératif s'est avant tout cristallisé autour d'un projet de réinvention des formes de la représentation agricole au niveau local, le motif avancé étant de mieux défendre les intérêts des agriculteurs en répondant de façon plus appropriée aux enjeux 
environnementaux par nature territorialisés. Ces organisations agricoles se sont appuyées sur un ancrage local fort; chacune d'elles s'est construite de façon plus ou moins étroite à partir d'un territoire agro-écologique donné ne pouvant s'étendre démesurément. La définition de ces territoires, en général présentée dans les textes fondateurs de chacune de ces organisations, prend en compte de façon plus ou moins explicite des dimensions agronomiques (types de sols, types de production, etc.), écologiques (éléments paysagers à entretenir, espèces botaniques ou ornithologiques à protéger, etc.) et économiques (activités agro-touristiques, produits agricoles de qualité, circuits courts, etc.). Elles ont, de ce fait, chacune adopté un nom emblématique de leur territoire leur permettant de les distinguer les unes des autres ${ }^{3}$. Du fait de cette volonté collective de s'unir localement face aux prescriptions réglementaires environnementales, ces organisations ont été qualifiées de "groupes de pression» (Polman, 2002, p. 137), de « contre-pouvoirs » locaux (Hagedorn et al., 2002, p. 18).

Ce renouvellement de la représentation agricole s'est articulé avec un projet plus global de représentation des intérêts du monde rural s'appuyant sur une stratégie d'ouverture du "territoire professionnel » 4 à des acteurs ruraux non agricoles (Daniel, 2011b). Certaines de ces organisations se sont, en effet, ouvertes aux sympathisants locaux non agricoles. Elles se sont présentées comme des plateformes locales permettant de prendre en compte la voix de certains acteurs ruraux dont les destins sont en partie liés à ceux du devenir de l'agriculture locale. Dans un contexte néerlandais où les discours sur la ruralité n'ont jamais été aussi divergents, les agriculteurs ont cherché des alliés pour faire valoir leurs intérêts et leurs propres visions du développement de l'agriculture. Les coopératives environnementales se sont structurées autour de nouveaux réseaux d'acteurs regroupant, entre autres, des organisations écologiques, des associations de propriétaires ou encore des entrepreneurs ruraux. Les coopératives ont tenté d'enrôler ces tiers non agricoles dans leur mouvement de revendication et sont, pour certaines d'entre elles, parvenues à les intégrer dans leur conseil d'administration. Cette ouverture a constitué, pour les agriculteurs, une stratégie visant à crédibiliser le mouvement, légitimer le rôle des agriculteurs dans l'entretien du territoire et surtout peser symboliquement sur la construction des normes environnementales. Elle leur a permis de jouer sur le double registre de la défense des intérêts du monde rural et de la représentation professionnelle.

Cette refonte en profondeur de la représentation des intérêts agricoles au niveau local a été favorisée par deux facteurs congruents. La profession agricole a, d'une part, été marquée par une érosion générale de l'influence de ses organisations dans la gestion des affaires agricoles. Cet affaiblissement a été à l'origine d'une transformation des relations entre les pouvoirs publics et le groupe professionnel. Il s'est matérialisé, au milieu des années 1990, par la dissolution du Landbouwschap, principal organe de cogestion des affaires rurales et agricoles entre l'Etat et la profession agricole aux

\footnotetext{
3 Par exemple, l'une de ces organisations se situant sur un îlot de terres sablonneuses au milieu d'un polder à dominante argileuse a pour nom « la rivière et la dune » (rivierduin en néerlandais). La dune fait ici référence aux terres sablonneuses et la rivière au paysage existant avant que le polder n'ait été mis en place.

${ }^{4}$ Cette expression est empruntée à Claude Dubar et Pierre Tripier (1998).
} 
Pays-Bas depuis 1954. Cette perte d'influence a incité les agriculteurs à réinventer de nouvelles façons de faire prévaloir plus efficacement leurs intérêts. Les dissensions émergentes au sein du groupe professionnel portant sur les réponses à apporter à la question environnementale à partir des années 1980 ont, d'autre part, poussé certains groupes d'agriculteurs à se tourner vers des solutions alternatives. Les positions radicales de responsables syndicaux niant la réalité des problèmes d'environnement n'ont en réalité cessé de mettre en lumière leur incapacité à ancrer leurs revendications dans la diversité et la complexité des territoires, et leur inefficacité à faire prévaloir les intérêts des agriculteurs. Les initiateurs des coopératives environnementales ont dès lors produit une réponse plus nuancée visant à articuler davantage les revendications des agriculteurs aux problématiques environnementales locales. Ils ont produit un discours plus adapté à leur territoire et, par la même occasion, se sont réapproprié, au niveau local, la représentation de leurs intérêts en tentant d'enrôler des alliés ruraux non agricoles.

Ce projet de refonte «agri-ruraliste» de la représentation - pour reprendre l'expression de Jaap Frouws (1998) - n'a toutefois pas eu tous les effets escomptés. Il a été montré par ailleurs que cette ouverture n'a pas été systématiquement rencontrée dans l'ensemble des coopératives et qu'elle n’a pas nécessairement donné naissance à des coalitions d'acteurs unanimement réunis autour de l'enjeu de défense du groupe agricole (Daniel, 2011b). Le projet de représentation est resté tourné autour d'un projet foncièrement agricole et, malgré les objectifs d'ouverture, la difficulté à harmoniser les motifs d'engagements des agriculteurs avec les aspirations - parfois critiques - des acteurs non agricoles a pérennisé des formes de solidarités quasi-exclusivement agricoles. Même en adhérant aux démarches des coopératives, les acteurs non agricoles ne se sont pas systématiquement alignés sur les revendications des agriculteurs et ont conservé une certaine distance avec le discours agricole. "Il y a des agriculteurs en qui je peux faire confiance (pour gérer la nature), mais il y en a d'autres, je suis sûr, ils ont un double agenda, vous voyez. Ils font ça pour des raisons économiques, pour l'argent ${ }^{5}$."

Si le projet coopératif n'a pas réussi à faire l'unanimité au sein des acteurs ruraux, il est toutefois parvenu à transformer la logique de segmentation du groupe professionnel. Alors que l'organisation syndicale était jusque dans les années 1980 organisée en "piliers » confessionnels ${ }^{6}$ (catholiques, protestants, libéraux), la création d'un syndicat unique appelé le Land-en Tuinbouworganisatie (LTO) à partir de 1995 a permis d'unifier ces différents piliers pour faire face à la perte générale d'influence du secteur. L'émergence des coopératives environnementales a eu pour effet de resegmenter le groupe, cette fois-ci autour des positions divergentes liées aux questions environnementales. La posture pragmatique des coopératives environnementales, à qui l'on reprochait d'être passées à l'ennemi, a été, dans un premier temps, stigmatisée par les responsables professionnels agricoles. Ce clivage, qui a marqué les premières années d'existences des coopératives environnementales, s'est en apparence atténué à partir de la fin des années 1990, lorsque le syndicat dominant LTO a finalement choisi

${ }^{5}$ Entretien avec un membre d'une organisation environnementale locale, le 14/05/2004.

6 Pour l'organisation de la société néerlandaise en « piliers », voir les travaux de Lijphart (1968). 
de reconnaître le mouvement et de s'approprier à son tour un discours proenvironnemental. Face aux risques de fragmentation de la représentation qui auraient pu être fatal à la profession dans sa globalité, le LTO s'est efforcé non seulement de reconnâtre le mouvement, mais aussi de le structurer au sein d'une fédération de coopératives aux niveaux national et régional ${ }^{7}$. Cette structure a permis aux responsables des coopératives de généraliser leur démarche et d'assurer au niveau national la représentation des territoires. Les dissensions entre agriculteurs adhérents aux coopératives environnementales et les autres plutôt hostiles n'ont toutefois pas disparu ${ }^{8}$. Les tensions entre les différents «segments professionnels»-au sens de Butcher et Strauss (1961) - se sont en fait déplacées au sein même de la structure syndicale. Ainsi, bien que les coopératives aient été rattachées aux organisations professionnelles plus traditionnelles, leur émergence a contribué à re-segmenter le groupe professionnel 9 .

\section{Les projets d'innovation ou « comment inventer une nouvelle agriculture?»}

Le projet coopératif s'est structuré de façon concomitante, au début des années 1990, autour de démarches d'innovation et d'expérimentations institutionnelles et techniques destinées à donner corps au projet d'autorégulation locale. Les fondateurs des premières organisations locales ont, à cette époque, proposé aux pouvoirs publics d'assouplir les réglementations environnementales qui mettaient en péril, selon eux, la viabilité de leurs exploitations agricoles. L'idée était qu'en échange de ces assouplissements, les coopératives s'engagent à expérimenter des pratiques agricoles innovantes plus ajustées à la diversité des territoires agro-écologiques. Les pouvoirs publics ont alors accordé de façon ponctuelle des marges de manœuvre aux agriculteurs et des expérimentations de terrains ont été mises en place pour tester les capacités des coopératives à atteindre par elles-mêmes les objectifs environnementaux.

Dans un premier temps, une expérimentation visant à mutualiser la gestion des pollutions agricoles s'est mise en place au début des années 1990. L'idée de ce projet était de substituer au principe pollueur-payeur une gestion plus flexible de la pollution en instituant des objectifs globaux pour une région donnée tout en laissant les agriculteurs libres de se coordonner pour réduire collectivement les pollutions. La

\footnotetext{
7 Dans le Nord Brabant notamment, il est même nécessaire d'adhérer au syndicat LTO pour devenir membre de ces organisations.

${ }^{8}$ Remarquons au passage que la segmentation du groupe professionnel néerlandais est différente de celle rencontrée en France, davantage structurée par les débats sur les "modèles » agricoles (Fouilleux, 2000).

${ }^{9}$ Il est très difficile de faire un lien entre cette segmentation et les caractéristiques objectives des structures d'exploitation adhérant aux coopératives, car ce type de données n'existe pas pour le moment. L'enquête réalisée par Oerlemans et al. (2004) a toutefois mis en évidence la diversité des profils d'exploitations membres. Si la petite exploitation laitière intensive semble majoritairement représentée dans $62 \%$ des cas, on retrouve également une majorité de céréaliers-cultivateurs dans près de $11 \%$ des organisations et une répartition proportionnelle entre différents types de producteurs dans $25 \%$ des coopératives.
} 
taxation globale de la région eût alors été fonction de la réalisation de ces objectifs régionaux indépendamment des contributions individuelles. L'objectif de cet arrangement institutionnel était de faire compenser les situations polluantes par celles où les apports en effluents d'élevage étaient moins importants afin de ne pas bloquer l'économie agricole locale. Ce projet a été, en fait, très vite abandonné du fait de son articulation difficile avec la Directive Nitrates.

C'est principalement, dans un second temps, via des projets visant à expérimenter et mutualiser des nouvelles pratiques et techniques agricoles plus respectueuses de l'environnement que des logiques d'expérimentation ont véritablement pris forme. Des projets se sont développés autour de réflexions techniques portant tout à la fois sur des changements de pratiques agricoles et des projets économiques alternatifs: agro-tourisme, produits agricoles régionaux de qualité ou encore agriculture biologique, etc. ${ }^{10}$ (Van der Ploeg et Renting, 2001). Dans la Province de Frise, par exemple, des expérimentations ont été menées dans le domaine de l'entretien des haies suite au retrait de l'application de la « loi ammoniac », votée en 1987, sur le territoire de la coopérative environnementale VEL. Cette loi qui limitait fortement les possibilités d'agrandissement des exploitations agricoles sur le territoire en proximité des zones identifiées comme « remarquable » d'un point de vue patrimonial a été, dans un premier temps, appliquée sur le territoire de VEL afin de protéger le paysage bocager typique de cette région. L'application de cette loi sur ce territoire menaçait paradoxalement ce paysage car si les exploitations venaient petit à petit à disparaître, c'est aussi la pérennité des haies et de leur entretien qui était menacée. En échange d'une annulation de la loi sur leur territoire, les agriculteurs se sont engagés dans des projets expérimentaux de restauration et d'entretien des haies afin de mieux connaître les éléments paysagers et d'identifier des pratiques d'entretien adaptées à une vision sophistiquée de ce paysage. Aux termes de ces expérimentations qui ont fait intervenir les autorités provinciales ainsi qu'une association locale de protection du paysage LBF (Landschapsbeheer Friesland) ${ }^{11}$, une catégorisation sophistiquée des différents types de haies a pu voir le jour (Daniel, 2005).

Ces dynamiques d'expérimentation ont pu émerger dans un contexte institutionnel marqué par une remise en cause profonde de l'organisation de la production et de la diffusion des connaissances en matière de développement agricole. Dans un contexte de crise du modèle sociotechnique de développement agricole, la privatisation du conseil agricole a créé un vide dans l'organisation et la diffusion du progrès technique. Ce vide a été toutefois comblé par un réaménagement des modes publics de financement de l'innovation en agriculture. Des logiques de projets, limités dans le temps, sur des problématiques de développement durable, financés tant par le ministère de l'Agriculture que par le ministère de l'Environnement ou les autorités locales, se sont substituées à l'organisation plus traditionnelle du conseil agricole,

10 Parmi les coopératives concernées par la mise en place d'expérimentations de terrain pendant les années 1990, on compte "Vel en Vanla » en Frise, «Milieu Cooperatie Peel » dans le Nord-Brabant, «Waterland» dans la province d'Hollande du Nord, «Ommer Marke te Ommen » dans la Province d'Overijssel, et la coopérative du sud Limbourg.

11 Landschapsbeheer Friesland signifie littérallement 'gestion du paysage en Frise'. 
fondée sur un modèle de diffusion plus structuré et homogène. Des opportunités ponctuelles de financement ont ainsi permis à certaines de ces coopératives de bénéficier de fonds publics et de marges de manœuvre significatives pour mener à bien leurs expérimentations.

Les formes de solidarités créées entre agriculteurs autour de ces expérimentations ont, dans certains cas, associé des acteurs extérieurs au monde agricole (organisations environnementales, autorités locales, organisations de préservation du paysage, etc.) et des experts, scientifiques, qui se sont inscrits dans la continuité des projets. Elles ont permis de mener à bien des projets innovants autour de problématiques sociotechniques diverses (entretien des haies, optimisation technique de la gestion des effluents d'élevage sur l'exploitation, etc.) et ont favorisé des formes d'apprentissages mutuels résultant de la rencontre entre ces différents acteurs ${ }^{12}$ (Eshuis et Stuiver, 2005). Ces démarches ont toutefois leurs limites. Certaines d'entre elles ont fait l'objet de controverses concernant l'objectivité et la scientificité des protocoles de recherches mis en œuvre ${ }^{13}$ - ce qui n'a pas contribué à crédibiliser le mouvement. De plus, ces projets d'innovation ne se sont pas généralisés sur l'ensemble du territoire. Les financements sont restés limités à quelques projets localisés sur des territoires pilotes, ce qui a fortement limité la multiplication de cette forme de solidarité à l'ensemble des coopératives environnementales - la mobilisation d'un collectif d'acteurs de l'innovation sur chaque micro-territoire se révélant peu opérationnelle. Enfin, ces projets d'innovation n'ont pas abouti à des arrangements pérennes d'auto-gouvernance puisqu'une fois les programmes terminés, les marges de manœuvre données aux agriculteurs dans l'application des réglementations environnementales ont disparu. Ces solidarités forgées autour de projets d'innovation n'ont donc concerné qu'une minorité d'organisations, limitant ainsi la capacité du mouvement à justifier techniquement auprès des pouvoirs publics leurs velléités politiques d'autorégulation locale.

\section{Le projet de gestion de l'environnement ou « comment contribuer à la gestion des biens publics environnementaux?"}

C'est en réalité principalement à travers leur contribution aux politiques de protection de la nature et aux opportunités offertes par l'émergence de différentes procédures de financement que les coopératives environnementales ont véritablement essaimé sur l'ensemble du territoire. L'adoption en 1997 du programme de gestion de la nature (Programma Beheer) compte parmi l'une des mesures phares des politiques néerlandaises de soutien à la multifonctionnalité de l'agriculture (Daniel, 2010). Dans le cadre de cette politique publique, les coopératives environnementales ont été vues par les

\footnotetext{
12 Selon Stuiver et al. (2003, p. 27), ces dynamiques peuvent être considérées comme des 'laboratoires de terrains', c'est-à-dire des "assemblages bétérogènes d'études scientifiques indépendantes opérées par plusieurs acteurs dans un champ scientifique donné, et qui -à l'instar des laboratoires babituels sont prémunis contre les interférences et les perturbations extérieures».

13 Des controverses ont notamment porté sur les méthodes de calcul du circuit de l'azote dans le sol dans le cas des expérimentations menées sur les coopératives de Vel et de Vanla en Frise.
} 
pouvoirs publics comme des organisations pouvant assurer la gestion de la biodiversité et du paysage sur des territoires agricoles -à l'instar d'autres associations de protection de la nature (comme l'organisation Natuurmonumenten) qui bénéficient de contrats de gestion pour entretenir des espaces naturels « remarquables ». Cette politique prévoyait de déléguer la gestion de biens publics agroenvironnementaux à des structures agricoles pouvant assurer une gestion efficace de ces espaces. Elle accordait un supplément financier à l'octroi de la subvention agroenvironnementale pour tout groupe organisé d'agriculteurs sur un territoire donné. Les coopératives environnementales se sont ainsi vu attribuer des missions de gestion de biens environnementaux (biodiversité, qualité de l'eau, paysage, etc.).

Ce modèle de mise en capacité des coopératives par les pouvoirs publics s'est inscrit dans un mouvement général de réforme managériale des administrations publiques (De Vries et Yesilkagit, 1999). Ces réformes qui ont instauré dans les différentes administrations publiques des principes d'efficacité, d'autonomisation, de responsabilisation et d'évaluation de différentes unités de travail se sont également appliquées aux relations entre les pouvoirs publics et la profession agricole. C'est dans le cadre de ce modèle managérial que les coopératives ont été assimilées à des organes gestionnaires liés à la puissance publique par une relation contractuelle et soumis à des exigences d'efficacité de fonctionnement. Ce modèle gestionnaire fait écho au modèle organisationnel présenté par les économistes néo-institutionnels pour qui cette forme d'organisation collective de la protection de la nature devait permettre la mise en place d'une organisation institutionnelle "efficace " limitant des coûts de transaction. Le projet coopératif s'est ainsi largement structuré autour du projet de gestion des instruments agro-environnementaux, comme le montre ce témoignage d'agriculteur: "Notre association a été créée en 2001. Il fallait avoir une structure associative pour pouvoir recevoir l'argent du gouvernement. Et puis nous sommes allés à la Province et on a pu recevoir de l'argent du programme européen Leader ${ }^{14}$ ». C'est à la fin des années 1990, au moment de l'essor des opportunités de financement, que le nombre de coopératives s'est accru considérablement puisqu'il est passé de neuf, en 1993, à près de 90, en 2004 (Oerlemans et al., 2004). La plupart des coopératives qui ont vu le jour à cette époque ont de ce fait un projet fondateur étroitement lié à la gestion des politiques rurales et agroenvironnementales ${ }^{15}$. Les agriculteurs se sont en fait approprié l'idée selon laquelle ils pouvaient gérer la nature par eux-mêmes: "Nous pouvons nous aussi entretenir la campagne, nous pouvons nous aussi entretenir le paysage. (...) Payez-nous et on le fera encore mieux qu'eux (les organisations environnementales) 16 ». Ils se sont approprié le «logos gestionnaire »(Boussard, 2008), c'est-à-dire l'ensemble des éléments de discours qui concourent à une administration rationnelle, efficace et organisée de l'action de protection de l'environnement.

14 Entretien réalisé avec un agriculteur, président d'une coopérative environnementale, le 27/07/2004.

15 Toutes les organisations que nous avons rencontrées et qui sont apparues à la fin des années 1990 ou au début des années 2000 ont suivi cette logique. C'est le cas des associations De Kop van NOP, Cö̈peratie Rivierduingebied, Zwartemeerdijk, ou encore Ecolana.

16 Entretien avec un employé de la Fédération régionale de coopératives environnementales BoerenNatuur NLTO, le 19/04/2004. 
La portée de cette mise en capacité reste toutefois considérablement limitée. En réalité, les coopératives n'ont reçu aucun mandat concernant l'organisation financière et économique de la politique au niveau local. Le gouvernement néerlandais avait pourtant projeté, dans les premières années de fonctionnement du programme de gestion, de leur allouer des enveloppes budgétaires et de leur laisser le soin de répartir les montants entre agriculteurs. En raison des règles de transparence de gestion des subventions européennes, la Commission européenne s'est opposée à une telle utilisation des fonds agroenvironnementaux et le gouvernement néerlandais a été contraint de mettre fin à ce type de fonctionnement pour pouvoir continuer à bénéficier du cofinancement européen. Les coopératives environnementales ont donc été davantage mobilisées sur des missions d'information et de sensibilisation des agriculteurs aux procédures d'octroi des aides et à l'évolution générale des réglementions. Bien que la multiplication de ces organisations sur l'ensemble du territoire national corresponde à une reconnaissance partielle de l'utilité des coopératives environnementales pour la collectivité, celles-ci se sont en définitive trouvées inféodées à un projet gouvernemental au sein duquel leur rôle a été d'accompagner localement les agriculteurs en les tenant informés de la réglementation et des opportunités offertes par l'engagement dans une démarche agroenvironnementale.

Cette révision «à la baisse » du projet coopératif n'est donc pas sans souligner les transformations du projet coopératif dont les objectifs se sont sensiblement déplacés d'une logique de résistance et d'opposition à la normalisation environnementale à une logique de collaboration des agriculteurs au bon fonctionnement de la politique de protection de la nature. En redéfinissant les contours de ce projet, les pouvoirs publics ont opéré une reconnaissance symbolique des formes de contestation agricole émergentes, sans pour autant laisser de marge de manœuvre significative aux coopératives dans la mise en ouvre d'un projet d'auto-gouvernance local (Daniel, 2010). Le projet initial de solidarité professionnelle fondé sur la contestation des normes décidées au niveau national s'est vu quelque peu vidé de sa substance au profit d'une solidarité induite par les autorités publiques visant à légitimer les normes environnementales auprès des agriculteurs. Cette appropriation gouvernementale du projet coopératif des agriculteurs a transformé la vocation initiale du mouvement; elle a contribué à promouvoir des formes de solidarités engendrées en partie par les réformes managériales.

\section{Conclusion-Discussion}

L'examen du mouvement agricole néerlandais de coopération environnementale montre que le renouvellement des formes de solidarités entre agriculteurs ne répond pas nécessairement à un ordre naturel où des organisations locales se seraient autoconstituées pour rendre optimale l'organisation sociale de la gestion de la nature. Comme le montre cet article, ces solidarités se sont incarnées dans une diversité de projets coopératifs dont les logiques de structuration peuvent être résumées en deux étapes. Dans un premier temps, au début des années 1990, ces projets ont consisté à mieux défendre les intérêts agricoles et à inventer localement de nouvelles formes d'agriculture. Ces premières expérimentations institutionnelles n'auraient pu voir le 
jour sans que n'interviennent certains facteurs conjoncturels propres au contexte néerlandais. Perte d'influence de la profession agricole à partir de la fin des années 1980, divergences internes à la profession sur le positionnement stratégique à adopter face au contexte de crise, transformation des modes de financement du conseil agricole sont autant de facteurs institutionnels ayant contribué à structurer les démarches des premières générations de coopératives. C'est toutefois, dans un deuxième temps, au travers d'un projet coopératif de gestion des instruments d'action publique de protection de l'environnement que les coopératives environnementales se sont véritablement généralisées sur le territoire néerlandais. La plupart d'entre elles se sont en effet créées suite aux opportunités de financement occasionnées par le développement des politiques agro-environnementales. La généralisation de ces organisations est intimement liée au projet gestionnaire initié par les pouvoirs publics découlant des réformes managériales des administrations publiques et à l'incorporation par les agriculteurs des attendus de ce projet. Bien que les trois logiques associatives (représentation, innovation, gestion) s'enchevêtrent les unes aux autres (plus qu'elles ne s'opposent), c'est bien au travers des logiques gestionnaires que le projet coopératif s'est imposé sur le territoire néerlandais au cours des années 2000. Les logiques d'innovation ne se sont incarnées que dans un nombre limité de dynamiques localisées et le projet de renouvellement de la représentation professionnelle au niveau local s'est, quant à lui, principalement recentré autour du syndicat dominant LTO. Malgré la faible portée du projet gestionnaire, la mise en œuvre des politiques de gestion de la nature a donc provoqué un renouvellement des solidarités locales entre professionnels agricoles par l'acculturation des esprits au «logos gestionnaire».

Cette inflexion dans ce mouvement de restructuration des solidarités entre professionnels ne peut être assimilée à un processus de déprofessionnalisation, entendu comme l'assujettissement total des agriculteurs aux autorités publiques et aux mécanismes de contrôle mis en place dans le cadre des instruments agroenvironnementaux. Elle a en réalité contribué à redistribuer les légitimités au sein de la profession - en faveur des agriculteurs favorables à une appropriation stratégique du discours pro-environnemental - plus qu'elle n'a fait disparaître à proprement parler le groupe professionnel. Cette recomposition traduit un recul de «l'autonomie professionnelle », c'est-à-dire de la capacité des professionnels à peser sur la définition de leur travail (Boussard et al., 2010); elle traduit une nouvelle forme de professionnalisme caractérisée par un accroissement considérable de l'emprise de l'administration sur le groupe professionnel. Si les premières coopératives environnementales se sont appuyées sur des démarches de développement rural " endogènes », " nées de l'intérieur » (born from within) (Van der Ploeg et Long, 1994), leur généralisation sur le territoire correspond à l'inverse à l'institutionnalisation d'un "professionnalisme de l'extérieur»(from above) (Evetts, 2006 ; Dubar et al., 2011) caractérisé par une substitution des valeurs gestionnaires aux valeurs professionnelles plus traditionnelles.

Cette recomposition du paysage professionnel s'incarne, paradoxalement, dans un projet de solidarité au service des cadres réglementaires contre lesquels les agriculteurs avaient lutté. Les nouvelles organisations agricoles sont devenues, pour la plupart, un des maillons de l'application des réglementations environnementales à l'origine de la 
mobilisation des initiateurs des premières coopératives. Le modèle de coopération s'est en cela subrepticement écarté du programme fort de résistance et de négociation qu'il s'était fixé initialement, au profit d'une démarche plus consensuelle d'adhésion et de participation à la politique gouvernementale - sans que les aspirations autorégulationnistes des agriculteurs aient pu véritablement aboutir. Cette recomposition " managériale » des solidarités entre professionnels agricoles entre en tension avec les aspirations initiales de résistance des agriculteurs, plus qu'elle ne s'y substitue. Certes, la deuxième génération de projets coopératifs qui a émergé à partir des années 2000 est moins marquée par le programme ambitieux des premières générations de coopératives, mais la possibilité d'élargissement de leur spectre d'action n'est pas à exclure. Bien au contraire, l'adhésion des agriculteurs à ces formes organisationnelles a contribué à structurer de nouvelles dynamiques d'agriculteurs, certes atomisées au niveau local, mais assurément plus aux prises techniquement et politiquement avec la complexité des enjeux environnementaux. Cette généralisation des coopératives environnementales fondée sur l'appropriation du logos gestionnaire n'est donc peut-être qu'une étape dans le processus de renégociation de la place et de la légitimité des agriculteurs néerlandais dans la gestion des affaires agricoles.

\section{Bibliographie}

Bezes P. (2008) Le tournant néomanagérial de l'administration française, in: Politiques publiques 1: la France dans la gouvernance européenne, Borraz O., Guiraudon V., Paris, Presses de Sciences Po, 215-255.

Boonstra W.J. (2006) Polder limits: A case study of value-conflicts on Dutch rural land use, $\mathrm{PhD}$ thesis, Wageningen University, $163 \mathrm{p}$.

Boussard V. (2008) Sociologie de la gestion. Les faiseurs de performance, Paris, Belin, 263 p.

Boussard V., Demazière D. et Milburn P. (éds) (2010) L'injonction au professionnalisme. Analyses d'une dynamique plurielle, Rennes, Presses universitaires de Rennes, 176 p.

Butcher R., Strauss A. (1961) Professions in process, American Journal of Sociology 66, 325-334.

Daniel F.-J. (2011a) Action research and performativity: How sociology shaped a farmers' movement in The Netherlands, Sociologia Ruralis 51, 17-34.

Daniel F.-J. (2011b) Environnement et contre-mobilisations agricoles. Vers une analyse des mouvements professionnels de résistance à la normalisation environnementale aux Pays-Bas, Etudes Rurales 187 (Janvier-Juin), 203-216.

Daniel F.-J. (2010) La « gestionnarisation » des politiques de protection de la nature aux Pays-Bas, Politix 23 (91), 159-177.

Daniel F.-J. (2005) Administering the multifunctionality of agriculture: A managerialist model in The Netherlands, Paper presented at the XXI Congress ESRS, Keszthely (Hungary). 
De Bruin R., Van Der Ploeg J.D. (1991) Keeping Track: Farming Styles and the Management of Nature and Landscape in the Northern Frisian Woodlands and the Southern Westerquarter, Wageningen, The Netherlands, Circle of Rural European Studies (In Dutch), 112 p.

De Visscher C., Varone F. (2004) La nouvelle gestion publique «en action », Revue internationale de politique comparée 11 (2), 177-185.

De Vries J., Yesilkagit K. (1999) Core executives and party policies: Privatization in The Netherlands, West European Politics 22 (1), 115-137.

Demailly L., de la Broise P. (2009) Les enjeux de la déprofessionnalisation, Socio-logos. Revue de l'association française de sociologie (4), http://socio-logos.revues.org/2305.

Demazière D., Gadéa C. (éds) (2009) Sociologie des groupes professionnels. Acquis récents, nouveaux défis, Paris, La Découverte, 463 p.

Deverre C., de Sainte Marie C. (2008) L'écologisation de la politique agricole européenne, Revue d'Etudes en Agriculture et Environnement 89 (4), 83-104.

Devienne S. (1989) Systèmes de production du lait et politique agricole aux Pays-Bas, Thèse de doctorat de l'Institut national agronomique Paris-Grignon, 489 p.

Dubar C., Tripier P. (1998) Sociologie des professions, Paris, Armand Colin, 256 p.

Dubar C., Tripier P. et Boussard V. (2011) Sociologie des professions, $3^{e}$ éd., Paris, Armand Colin, collection « Collection U », 369 p.

Durkheim E. (2007 [1893]) De la division du travail social, Paris, PUF, 416 p.

Eshuis J., Stuiver M. (2005) Creating situated knowledge through joint learning processes among dairy farmers and scientists in a mineral project in The Netherlands, Agriculture and Human Values 22 (2), 137-148.

Evetts J. (2006) Introduction. Trust and professionalism: Challenges and occupational changes, Current Sociology 54 (4), 515-531.

Fouilleux E. (2000) Entre production et institutionnalisation des idées, la réforme de la Politique agricole commune, Revue française de Science politique 50 (2), 277-305.

Frouws J. (1998) The contested redefinition of the countryside. An analysis of rural discourses in The Netherlands, Sociologia Ruralis 38 (1), 54-68.

Hagedorn K., Arzt K. and Peters U. (2002) Institutional arrangements for environmental co-operatives: A conceptual framework, in: Environmental Cooperation and Institutional Change: Theories and Policies for European Agriculture, Hagedorn K., Cheltenham, Edward Elgar, 3-25.

Hervieu B., Lagrave R.-M. (éds) (1992) Les syndicats agricoles en Europe, Paris, L'Harmattan, collection Alternatives rurales, $318 \mathrm{p}$.

Kalaora B. (2001) A la conquête de la pleine nature, Ethnologie française XXXVII (2), 591-597. 
Labarthe P. (2006) La privatisation du conseil agricole en question. Evolutions institutionnelles et performances des services de conseil dans trois pays européens (Allemagne, France, Pays-Bas), Thèse de doctorat en sciences économiques, Université de Marne-la-Vallée, 405 p.

Lijphart A. (1968) The Politics of Accommodation: Pluralism and Democracy in The Netherlands, Berkeley, University of California Press, 222 p.

Luttik J., Van der Ploeg B. (2004) Functions of agriculture in urban society in The Netherlands, in: Sustaining Agriculture and the Rural Environment: Governance, Policy and Multifunctionality, Brouwer F., Cheltenham (UK), Edward Elgar Publishing Limited, 204-222.

Oerlemans N., Van Well E., Guldemond J.A. (2004) Agrarische natuurverenigingen aan de slag, Achtergronddocument bij Natuurbalans, Wageningen, Natuurplanbureau, $40 \mathrm{p}$.

Padt F.J.G. (2007) Green Planning. An Institutional Analysis of Regional Environmental Planning in The Netherlands, Delft, Eburon, 272 p.

Pollitt C., Bouckaert G. (2000) Public Management Reform. A Comparative Analysis, Oxford, Oxford University Press, 314 p.

Polman N. (2002) Institutional Economics Analysis of Contractual Arrangements; Managing Wildlife and Landscape on Dutch Farm, PhD-thesis, Wageningen University, $198 \mathrm{p}$.

Polman N., Slangen L.H.G. (2002) Self-organizing and self-governing of environmental co-operatives: Design principles, in: Environmental Co-operation and Institutional Change: Theories and Policies for European Agriculture, Hagedorn K., Northampton, Edward Elgar, 91-111.

Slangen L.H.G., Polman N. (2002) Environmental co-operatives: A new institutional arrangement of farmers, in: Environmental Co-operation and Institutional Change: Theories and Policies for European Agriculture, Hagedorn K., Northampton, Edward Elgar, 69-90.

Stuiver M., Van der Ploeg J.D., et al. (2003) The VEL and VANLA environmental cooperatives as field laboratories, NJAS Wageningen Journal of Life Sciences 51 (1-2), 27-39.

Van der Ploeg J.D. (2003) The Virtual Farmer, Assen, Royal Van Gorcum, 432 p.

Van der Ploeg J.D., Long A. (eds) (1994) Born from Within. Practices and Perspectives of Endogenous Rural Development, Assen, Royal Van Gorcum, 298 p.

Van der Ploeg J.D., Renting H. (2001) Re-connecting nature, farming and society. Environmental co-operatives in The Netherlands as an institutional arrangement for creating coherence, Journal of Environmental Policy and Planning 3 (2), 85-101.

Zoll R. (1998) Le défi de la solidarité organique: avons-nous besoin de nouvelles institutions pour préserver la cohésion sociale? Sociologie et sociétés 30 (2), 1-10. 